\title{
On the Use of the Borel-Cantelli Lemma in Markov Chains
}

\author{
Alexei Stepanov, ${ }^{*}$ Immanuel Kant Baltic Federal University, Russia
}

\begin{abstract}
In the present paper, we propose technical generalizations of the Borel-Cantelli lemma. These generalizations can be further used to derive strong limit results for Markov chains. In our work, we obtain some strong limit results.
\end{abstract}

Keywords and Phrases: Markov chains; the Borel-Cantelli lemma; strong limit laws; the concomitants of order statistics; the $F^{\alpha}$-scheme.

AMS 2000 Subject Classification: 60F99, 60F15.

\section{Introduction}

Suppose $A_{1}, A_{2}, \cdots$ is a sequence of events on a common probability space and that $A_{i}^{c}$ denotes the complement of event $A_{i}$. The Borel-Cantelli lemma, presented here as Lemma 1.1, is used for producing strong limit theorems.

Lemma 1.1. 1. If, for any sequence $A_{1}, A_{2}, \cdots$ of events, $\sum_{n=1}^{\infty} P\left(A_{n}\right)<\infty$, then $P\left(A_{n}\right.$ i.o. $)=0$, where $i . o$. is an abbreviation for "infinitively often".

2. If $A_{1}, A_{2}, \cdots$ is a sequence of independent events and if $\sum_{n=1}^{\infty} P\left(A_{n}\right)=\infty$, then $P\left(A_{n}\right.$ i.o. $)=1$.

The first part of the Borel-Cantelli lemma is generalized in Barndorff-Nielsen (1961) and Balakrishnan and Stepanov (2010). These results are presented below as Lemma 1.2 and Lemma 1.3, respectively.

Lemma 1.2. Let $A_{1}, A_{2}, \ldots$ be a sequence of events such that $P\left(A_{n}\right) \rightarrow 0$. If $\sum_{n=1}^{\infty} P\left(A_{n} A_{n+1}^{c}\right)<\infty$, then $P\left(A_{n}\right.$ i.o. $)=0$.

*Immanuel Kant Baltic Federal University, A.Nevskogo 14, Kaliningrad, 236041 Russia; e-mail: alexeistep45@mail.ru 
Lemma 1.3. Let $A_{1}, A_{2}, \ldots$ be a sequence of events such that $P\left(A_{n}\right) \rightarrow 0$. If, for some $m \geq 0, \sum_{n=1}^{\infty} P\left(A_{n}^{c} \ldots A_{n+m-1}^{c} A_{n+m}\right)<\infty$, then $P\left(A_{n}\right.$ i.o. $)=0$.

Many publications were devoted to the second part of the Borel-Cantelli lemma in attempts to weaken the independence condition. Erdös and Rényi (1959) discovered that this condition can be replaced by the weaker condition of pairwise independence of events $A_{1}, A_{2}, \ldots$ They also found that the later condition, in its turn, can be replaced by the condition: $P\left(A_{i} A_{j}\right) \leq P\left(A_{i}\right) P\left(A_{j}\right)$ for every $i \neq j$. Further generalizations of the BorelCantelli lemma were obtained independently by Kochen and Stone (1964) and Spitzer (1964). Lamperti (1963) formulated the following proposition. If $C$ is a positive constant, $\sum_{n=1}^{\infty} P\left(A_{n}\right)=\infty$ and $P\left(A_{i} A_{j}\right) \leq C P\left(A_{i}\right) P\left(A_{j}\right)$ for all large enough $i \neq j$, then $P\left(A_{n}\right.$ i.o. $)>0$. Petrov (2002) showed that if $C \geq 1$, then $P\left(A_{n}\right.$ i.o. $) \geq 1 / C$. Recently Frolov (2012) extended the results of Petrov (2002), (2004) and found more sophisticated lower bounds for $P\left(A_{n}\right.$ i.o. $)$. The second part of the Borel-Cantelli lemma was also discussed in the works of Chang and Erdös (1952), Kounias (1968), Móri and Székely (1983), Martikainen and Petrov (1990), and Petrov (1995). For a review on the Borel-Cantelli lemma, one may refer to the book of Chandra (2012).

An alternative way to get around the independence assumption in the second part of the Borel-Cantelli lemma is to use conditioning, as was proposed in Lévy (1937).

Lemma 1.4. Let $A_{1}, A_{2}, \ldots$ be a sequence of events and $I_{A_{n}}$ the indicator function of the event $A_{n}$. Then with probability one

$$
\sum_{n=1}^{\infty} I_{A_{n}}=\infty \quad \text { iff } \quad \sum_{n=2}^{\infty} P\left(A_{n} \mid \sigma\left\{A_{1}, \ldots, A_{n-1}\right\}\right)=\infty
$$

Lévy's lemma implies the classical form of the Borel-Cantelli lemma.

In our work, we are going to formulate a version of the Borel-Cantelli lemma which is applicable to Markov chains. Let us define the sequences of events with Markov property.

Definition 1.1. We say that $A_{n}(n \geq 1)$ is a Markov sequence of events if the sequence of random variables $I_{A_{n}}(n \geq 1)$ is a Markov chain.

Obviously, Markov sequences of events are associated with Markov chains.

The rest of our paper is organized as follows. In Section 2, we present technical generalizations of the Borel-Cantelli lemma. These generalizations are stated in terms of Markov sequences of events and can be further applied to the asymptotic theory of Markov chains. In Section 3, the results of Section 2 are used to derive strong limit results for Markov chains. 


\section{The Borel-Cantelli Lemma for Markov Sequences of Events}

In this section, we consider Markov sequences of events and discuss conditions for the validity of $P\left(A_{n}\right.$ i.o. $)=0 / 1$. It is easy to show that the condition $P\left(A_{n}\right) \not \rightarrow 0$ implies that $P\left(A_{n}\right.$ i.o. $)>0$. In the following generalization of the Borel-Cantelli lemma we assume that $P\left(A_{n}\right) \rightarrow 0$.

Lemma 2.1. Let $A_{1}, A_{2}, \ldots$ be a Markov sequence of events such that $P\left(A_{n}\right) \rightarrow 0$, and $N$ be a number such that $P\left(A_{n}\right) \neq 1$ for $n \geq N$. Let us consider the series

$$
\sum_{n=N}^{\infty} P\left(A_{n+1} \mid A_{n}^{c}\right) .
$$

If the series in (2.1) is convergent, then $P\left(A_{n}\right.$ i.o. $)=0$. If the series in (2.1) is divergent, then $P\left(A_{n}\right.$ i.o. $)=1$.

Proof Let us choose $n \geq N$. It follows from the Markov property that

$$
\begin{gathered}
1-P\left(A_{n} \cup A_{n+1} \cup \ldots \cup A_{n+k}\right)=P\left(A_{n}^{c} A_{n+1}^{c} \ldots A_{n+k}^{c}\right)= \\
P\left(A_{n}^{c}\right) P\left(A_{n+1}^{c} \mid A_{n}^{c}\right) \ldots P\left(A_{n+k}^{c} \mid A_{n+k-1}^{c}\right) .
\end{gathered}
$$

Then

$$
1-P\left(\cup_{i=n}^{\infty} A_{i}\right)=e^{\log P\left(A_{n}^{c}\right)+\sum_{i=n}^{\infty} \log P\left(A_{i+1}^{c} \mid A_{i}^{c}\right)} .
$$

Since $\log (1-x) \sim-x$ as $x \rightarrow 0$, the convergence/divergence of the series $\sum_{i=n}^{\infty} P\left(A_{i+1} \mid A_{i}^{c}\right)$ implies the convergence/divergence of the series $\sum_{i=n}^{\infty} \log P\left(A_{i+1}^{c} \mid A_{i}^{c}\right)$ (and vice verse). Observe that $P\left(A_{n}\right.$ i.o. $)=\lim _{n \rightarrow \infty} P\left(\cup_{i=n}^{\infty} A_{i}\right)$. The result of Lemma 2.1 readily follows from (2.2).

Remark 2.1. It should be noted that the first statement in Lemma[2.1 can be derived from Lemma 1.3 and the second statement in Lemma 2.1 can be obtained from Lemma 1.4.

Since $P\left(A_{n+1} \mid A_{n}^{c}\right)=\frac{P\left(A_{n}^{c} A_{n+1}\right)}{P\left(A_{n}^{c}\right)}$ and in Lemma $2.1 P\left(A_{n}^{c}\right) \rightarrow 1$, one can rewrite Lemma 2.1 in the following way.

Lemma 2.2. Let $A_{1}, A_{2}, \ldots$ be a Markov sequence of events such that $P\left(A_{n}\right) \rightarrow 0$. Let us consider the series

$$
\sum_{n=1}^{\infty} P\left(A_{n}^{c} A_{n+1}\right) .
$$

If the series in (2.4) is convergent, then $P\left(A_{n}\right.$ i.o. $)=0$. If the series in (2.4) is divergent, then $P\left(A_{n}\right.$ i.o. $)=1$. 
Obviously, if the Markov property holds for some sequence $A_{n}$, then

$$
P\left(A_{n}^{c} A_{n+1}^{c} \ldots A_{n+k}^{c}\right)=P\left(A_{n}^{c} \mid A_{n+1}^{c}\right) \ldots P\left(A_{n+k-1}^{c} \mid A_{n+k}^{c}\right) P\left(A_{n+k}^{c}\right) .
$$

With little modifications in the above proofs, one can show the following result.

Lemma 2.3. Let $A_{1}, A_{2}, \ldots$ be a Markov sequence of events such that $P\left(A_{n}\right) \rightarrow 0$. Let us consider the series

$$
\sum_{n=1}^{\infty} P\left(A_{n} A_{n+1}^{c}\right)
$$

If the series in (2.4) is convergent, then $P\left(A_{n}\right.$ i.o. $)=0$. If the series in (2.4) is divergent, then $P\left(A_{n}\right.$ i.o. $)=1$.

We present some further simple generalizations of the Borel-Cantelli lemma which are applicable to the theory of Markov chains. It is known that a sequence $X_{1}, X_{2}, \ldots$ forms a Markov chain of order $k$ (with memory $k$ ) if for all $n>k$

$$
P\left(X_{n}=x_{n} \mid X_{n-1}=x_{n-1}, \ldots, X_{1}=x_{1}\right)=P\left(X_{n}=x_{n} \mid X_{n-1}=x_{n-1}, \ldots, X_{n-k}=x_{n-k}\right) .
$$

Definition 2.1. We say that $A_{n}(n \geq 1)$ is a Markov sequence of events of order $k$ if the sequence of random variables $I_{A_{n}}(n \geq 1)$ is a Markov chain of order $k$.

Lemma 2.1 and Lemma 2.2 can be easily extended to such sequences.

Lemma 2.4. Let $A_{1}, A_{2}, \ldots$ be a Markov sequence of events of order $k$ such that $P\left(A_{n}\right) \rightarrow 0$, and $N$ be a number such that $P\left(A_{n}\right) \neq 1$ for $n \geq N$. Let us consider the series

$$
\sum_{n=\max \{k, N\}}^{\infty} P\left(A_{n+1} \mid A_{n}^{c} \ldots A_{n-k+1}^{c}\right) .
$$

If the series in (2.5) is convergent, then $P\left(A_{n}\right.$ i.o. $)=0$. If the series in (2.5) is divergent, then $P\left(A_{n}\right.$ i.o. $)=1$.

Lemma 2.5. Let $A_{1}, A_{2}, \ldots$ be a Markov sequence of events of order $k$ such that $P\left(A_{n}\right) \rightarrow 0$. Let us consider the series

$$
\sum_{n=1}^{\infty} P\left(A_{n}^{c} \ldots A_{n+k-1}^{c} A_{n+k}\right) .
$$

If the series in (2.6) is convergent, then $P\left(A_{n}\right.$ i.o. $)=0$. If the series in (2.6) is divergent, then $P\left(A_{n}\right.$ i.o. $)=1$.

\section{Applications}

In this section, we discuss two applications to the results of Section 2. In Subsection 3.1, we derive a strong limit theorem for the concomitants of maxima. In Subsection 3.2, we obtain strong limit results for the $F^{\alpha}$-scheme. 


\subsection{Strong Limit Results for the Concomitants of Maxima}

Assume in this subsection that $(X, Y),\left(X_{1}, Y_{1}\right),\left(X_{2}, Y_{2}\right), \ldots,\left(X_{n}, Y_{n}\right)$ are independent and identically distributed random vectors with continuous bivariate distribution function $F(x, y)$ and corresponding marginal distributions functions $H(x)$ and $G(y)$. In the case of existence the bivariate density of $(X, Y)$ will be denoted as $f(x, y)$. Let $X_{1, n} \leq X_{2, n} \leq \ldots \leq X_{n, n}$ be the order statistics obtained from the sample $X_{1}, X_{2}, \ldots, X_{n}$ and $Y_{[1, n]}, Y_{[2, n]}, \ldots, Y_{[n, n]}$ the corresponding concomitants of these order statistics, which relate to the sample $Y_{1}, Y_{2}, \ldots, Y_{n}$. We will also use the designations $Z_{[1]}=\left(X_{1,1}, Y_{[1,1]}\right), \ldots, Z_{[n]}=\left(X_{n, n}, Y_{[n, n]}\right)$. The concept of concomitants of order statistics is introduced in David (1973) and Bhattacharya (1974).

The limit behavior of $P\left(Y_{[n-k, n]} \leq y\right)$ when $k \geq 0$ is fixed and $n \rightarrow \infty$ is studied in many research works, see for example, David and Galambos (1974), (1987), Egorov and Nevzorov (1984), David (1994), Goel and Hall (1994), and David and Nagaraja (2003); see also the references therein.

Strong limit results for the concomitants of order statistics are rarely discussed. One can mention the publication of Goel and Hall (1994), where some strong limit theorems for differences between order statistics and concomitants are obtained, and the work of Sen (1981), in which the strong invariance principle for concomitants is discussed. In this subsection, to show the power of Lemma 2.2, we establish a new strong limit theorem for $Y_{[n, n]}$. This result can be further extended to the concomitants of top order statistics.

Let $l_{H}=\inf \{x \in \mathbb{R}: H(x)>0\}$ and $r_{H}=\sup \{x \in \mathbb{R}: H(x)<1\}$ be the left and right extremities of $H$, respectively.

In the absolutely continuous case, when the bivariate density $f(x, y)$ exists, one can write the joint density of $Z_{[1]}, \ldots, Z_{[n]}$ as

$$
f_{Z_{[1]}, \ldots, Z_{[n]}}\left(x_{1}, y_{1}, \ldots, x_{n}, y_{n}\right)=f\left(x_{1}, y_{n}\right) \ldots f\left(x_{n}, y_{n}\right) \quad\left(x_{1} \leq \ldots \leq x_{n}, y_{i} \in \mathbb{R}\right) .
$$

It follows that

$$
f_{Z_{[n+1]} \mid Z_{[n]} \ldots Z_{[1]}}\left(x_{n+1}, y_{n+1} \mid x_{n}, y_{n}, \ldots, x_{1}, y_{1}\right)=f\left(x_{n+1}, y_{n+1}\right) \quad\left(x_{n+1} \geq x_{n}, y_{n}, y_{n+1} \in \mathbb{R}\right) .
$$

The last equality implies that the sequence $Z_{[1]}, Z_{[2]}, \ldots$ forms a Markov chain. The Markov property also holds in the continuous case.

The following limit

$$
\lim _{x \rightarrow r_{H}} \frac{G(y)-F(x, y)}{1-H(x)}=\beta(y) \in[0,1]
$$

is considered in Bairamov and Stepanov (2010), (2011). They showed that if $\beta(y)=0$ for any $y<r_{G}=\sup \{y \in \mathbb{R}: G(y)<1\}$, then

$$
Y_{[n-k, n]} \stackrel{p}{\rightarrow} r_{G} \quad(n \rightarrow \infty) .
$$

With some additional conditions on $F$ one can formulate a strong limit law for $Y_{[n, n]}$. 
Theorem 3.1. The convergence

$$
Y_{[n, n]} \stackrel{a . s .}{\rightarrow} r_{G} \quad(n \rightarrow \infty)
$$

holds true iff

$$
\int_{\mathbb{R}} \frac{G(y)-F(x, y)}{(1-H(x))^{2}}[d H(x)-F(d x, y)]<\infty
$$

for any $y<r_{G}$.

Proof By symmetry and independence, we have

$$
\begin{gathered}
P\left(A_{n}\right)=P\left(Y_{[n, n]} \leq y\right)= \\
n \int_{\mathbb{R}} \int_{l_{G}}^{y} P\left(X_{2} \leq x, \ldots, X_{n} \leq x\right) F(d x, d v) .
\end{gathered}
$$

It follows that

$$
P\left(Y_{[n, n]} \leq y\right)=n \int_{\mathbb{R}} H^{n-1}(x) F(d x, y)
$$

By the argument that is used for obtaining (3.3), one can get

$$
\begin{gathered}
P\left(A_{n}^{c} A_{n+1}\right)=P\left(Y_{[n, n]}>y, Y_{[n+1, n+1]} \leq y\right)= \\
n \int_{\mathbb{R}} H^{n-1}(x)(G(y)-F(x, y))[d H(x)-F(d x, y)] .
\end{gathered}
$$

Then

$$
\begin{gathered}
\sum_{n=1}^{\infty} P\left(A_{n}^{c} A_{n+1}\right)= \\
\int_{\mathbb{R}} \frac{G(y)-F(x, y)}{(1-H(x))^{2}}[d H(x)-F(d x, y)] .
\end{gathered}
$$

Theorem 3.1 readily follows from Lemma 2.2 .

Remark 3.1. Observe that under condition (3.2) we have $\beta(y)=0$.

\subsection{Strong Limit Results for the $F^{\alpha}$-Scheme}

Let in the following, $X_{1}, X_{2}, \ldots, X_{n}$ be independent continuous random variables with distribution functions $F^{\alpha_{1}}, F^{\alpha_{2}}, \ldots, F^{\alpha_{n}}$, where $\alpha_{i}>0(i \geq 2)$ and $\alpha_{1}=1$. These settings are known as the $F^{\alpha}$-scheme, in which the independent variables $X_{1}, X_{2}, \ldots, X_{n}$ have the same support. When $\alpha_{i}=1$, the $F^{\alpha}$-scheme reduces to the case when the variables $X_{i}$ are independent and identically distributed. The $F^{\alpha}$-scheme was first considered by Yang (1975) as a 
model of a non-stationary sequence of independent random variables. Later it was discussed by Nevzorov (1985, 1986), Pfeifer (1989, 1991), Bairamov and Stepanov (2013), and others.

Strong Limit Results for Maxima Let in the following, $M_{n}=\max \left\{X_{1}, \ldots, X_{n}\right\}$, $A_{n}=1+\alpha_{2}+\ldots+\alpha_{n}$ and $x_{n}$ be a nondecreasing sequence of real numbers. It is easily found that $P\left(M_{n} \leq x_{n}\right)=F^{A_{n}}\left(x_{n}\right)$. It follows from the Borel-Cantelli lemma that if

$$
\sum_{n=1}^{\infty} F^{A_{n}}\left(x_{n}\right)<\infty
$$

then $P\left(M_{n} \leq x_{n}\right.$ i.o. $)=0$. One can show that the sequence $M_{n}(n \geq 1)$ forms a Markov chain. Lemma 2.3 allows us to formulate the following result.

Proposition 3.1. The equality

$$
P\left(M_{n} \leq x_{n} \text { i.o. }\right)=\left\{\begin{array}{l}
0 \\
1
\end{array}\right.
$$

holds true iff

$$
\sum_{n=1}^{\infty} F^{A_{n}}\left(x_{n}\right)\left[1-F^{\alpha_{n+1}}\left(x_{n+1}\right)\right]\left\{\begin{array}{l}
<\infty \\
=\infty .
\end{array}\right.
$$

Observe that if $F^{\alpha_{n}}\left(x_{n}\right) \rightarrow 1$, then the series in (3.5) converges under weaker conditions than the serious in (3.4). One can propose examples when the Borel-Cantelli lemma fails to produce strong limit results and Lemma 2.3 produces strong limit results in forms of "iff" statements. One of such examples is given below.

Example 3.1. Let $F$ be the unit uniform distribution. Let us choose

$$
x_{n}=1-\frac{\log \log n}{n}, \quad \alpha_{n}=\gamma(1+1 / n) \quad \text { and } \quad A_{n} \approx \gamma(n+\log n) .
$$

where $\gamma>0$. Observe that $F^{A_{n}}\left(x_{n}\right) \rightarrow 0$ and $\sum_{n=1}^{\infty} F^{A_{n}}\left(x_{n}\right)=\infty$ for any $\gamma>0$. It follows that the Borel-Cantelli lemma can not help us in this case.

The series $\sum_{n=1}^{\infty} F^{A_{n}}\left(x_{n}\right)\left[1-F^{\alpha_{n+1}}\left(x_{n+1}\right)\right]$ behaves like the series $\sum_{n=1}^{\infty} \frac{\log \log n}{n(\log n)^{\gamma}}$ when $\gamma>0$. Lemma 2.3 then states that

$$
P\left(M_{n} \leq 1-\frac{\log \log n}{n} \text { i.o. }\right)=\left\{\begin{array}{l}
0 \\
1
\end{array}\right.
$$

iff

$$
\gamma \in\left\{\begin{array}{c}
(1, \infty) \\
(0,1]
\end{array}\right.
$$


Could the newcomer be a maximum? Observe that if $X \sim F, Y \sim F^{\alpha}$ and $\alpha>1(0<\alpha<1)$, then $Y \geq_{s t} X\left(Y \leq_{s t} X\right)$, where st means the stochastic comparison of $X$ and $Y$. That way, choosing $\alpha_{n}$ as an increasing sequence for the $F^{\alpha}$-scheme, we shift probability masses $F^{\alpha_{n}}$ towards the right end of the common support. This gives new members of the sequence $X_{1}, \ldots, X_{n}, X_{n+1}$ (we address to $X_{n+1}$ as to a new member) better chances to become maxima. We will show that for properly chosen $\alpha_{n}$ and all large enough $n$ each consecutive sample observation can be a maximum. On the contrary, choosing the sequence $\alpha_{n}$ as decreasing we can "prohibit" newcomers to be maxima.

Suppose that $\alpha_{n} \rightarrow \infty$, which means that $A_{n} \rightarrow \infty$. As was pointed out, under the proper rate of increase of $\alpha_{n}$, each new sample observation can be a maximum. The corresponding conditions are proposed below in Proposition 3.2 and Remark 3.2.

Proposition 3.2. Let $\alpha_{n} \rightarrow \infty$ and $A_{n} / A_{n+1} \rightarrow 0$. Then

$$
P\left(M_{n}-X_{n}>0 \text { i.o. }\right)=\left\{\begin{array}{l}
0 \\
1
\end{array}\right.
$$

iff

$$
\sum_{n=1}^{\infty} A_{n} / A_{n+1}\left\{\begin{array}{l}
<\infty \\
=\infty
\end{array}\right.
$$

Proof Let us denote $B_{n}=\left\{M_{n}-X_{n}>0\right\}$ and $B_{n}^{c}=\left\{M_{n}=X_{n}\right\}$. Then

$$
P\left(B_{n}\right)=\int_{\mathbb{R}} F^{\alpha_{n}}(x) d F^{A_{n-1}}(x)=A_{n-1} / A_{n}
$$

and

$$
\begin{aligned}
P\left(B_{n} B_{n+1}^{c}\right)= & \int_{\mathbb{R}} F^{\alpha_{n}}(x)\left(1-F^{\alpha_{n+1}}(x)\right) d F^{A_{n-1}}(x)= \\
& \frac{\alpha_{n+1}}{A_{n+1}} \cdot \frac{A_{n-1}}{A_{n}} \sim \frac{A_{n-1}}{A_{n}} .
\end{aligned}
$$

The result follows from Lemma 2.3 , (3.7) and (3.8).

Remark 3.2. If the series in (3.6) is convergent, then $M_{n}-X_{n} \rightarrow 0$ a.s., i.e. for all large enough $n$ with probability one each new sample member is a maximum. This, in particular, happens when $\alpha_{n}=n^{2 n}$.

As was mentioned above, a properly chosen $\alpha_{n}$ can "prohibit" newcomers to be maxima. This issue is discussed in the rest of our work.

Proposition 3.3. Let

$$
\alpha_{n} / A_{n} \rightarrow 0
$$


Then

iff

$$
P\left(M_{n}=X_{n} \text { i.o. }\right)=\left\{\begin{array}{l}
0 \\
1
\end{array}\right.
$$

$$
\sum_{n=1}^{\infty} \alpha_{n} / A_{n}\left\{\begin{array}{l}
<\infty \\
=\infty
\end{array}\right.
$$

Proof The proof is similar to the proof of Proposition 3.2 . One should take $C_{n}=B_{n}^{c}, C_{n}^{c}=$ $B_{n}$ and apply Lemma 2.3 to the sequence of events $C_{n}$.

Remark 3.3. If the series in (3.10) is convergent, then $M_{n}>X_{n}$ a.s., i.e. for all large enough $n$ with probability one no new sample member can be a maximum. This, in particular, happens when $\alpha_{n}=n^{-2}$.

\section{References}

Bairamov, I. and Stepanov, A. (2010). Numbers of near-maxima for the bivariate case, Statistics Probability Letters, 80 (3), 196-205.

Bairamov, I. and Stepanov, A. (2011). Numbers of near bivariate record-concomitant observations spacings, Journal of Multivariate Analysis, 102 (5), 908-917.

Bairamov, I. and Stepanov, A. (2013). Numbers of near-maxima for $F^{\alpha}$-scheme, Statistics, 47, 191-201.

Balakrishnan, N., Stepanov, A. (2010). Generalization of Borel-Cantelli lemma - The Mathematical Scientist, 35 (1), 61-62.

Barndorff-Nielsen, O. (1961). On the rate of growth of the partial maxima of a sequence of independent identically distributed random variables. Math. Scand., 9, 383-394.

Bhattacharyya, B. B. (1974). Convergence of sample paths of normalized sums of induced order statistics, Ann. Statist., 2, 1034-1039.

Chung, K.L. and Erdös, P. (1952). On the application of the Borel-Cantelli lemma. Trans. Amer. Math. Soc., 72, 179-186.

Chandra, T.K. (2012). The Borel-Cantelli Lemma, Springer Briefs in Statistics.

David, H. A. (1973). Concomitants of order statistics, Bull. Inst. Internat. Statist., 45 (1), 295-300.

David, H. A. and Galambos, J. (1974). The asymptotic theory of concomitants of order statistics, J. Appl. Prob., 11, 762-770. 
David, H.A., Nagaraja, H.N. (2003). Order Statistics. Third edition, John Wiley \& Sons, New York.

Egorov, V. A. and Nevzorov, V. B. (1984). Rate of Convergence to the Normal Law of Sums of Induced Order Statistics, Journal of Soviet Mathematics (New York), 25, 1139- 1146.

Erdös, P. and Rényi, A. (1959). On Cantor's series with convergent $\sum 1 / q_{n}$. Ann. Univ. Sci. Budapest. Sect. Math., 2, 93-109.

Frolov, A.N. (2012). Bounds for probabilities of unions of events and the Borel-Cantelli lemma. Statist. Probab. Lett., 82, 2189-2197.

Goel, P.K. and Hall, P. (1994). On the average difference between concomitants and order statistics, The Annals of Probability, 22 (1), 126-144.

Kochen, S.B. and Stone, C.J. (1964). A note on the Borel-Cantelli lemma. Illinois J. Math., 8, 248-251.

Kounias, E.G. (1968). Bounds for the probability of a union, with applications. Ann. Math. Statist., 39, 2154-2158.

Lamperti, J. (1963). Wieners test and Markov chains. J.Math.Anal.Appl., 6, 58-66.

Lévy, P. (1937). Theorie de l addition des variables aleatoires, Gauthier-Villars, Paris.

Martikainen, A.I., Petrov, V.V., (1990). On the BorelCantelli lemma. Zapiski Nauch. Semin. Leningrad. Otd. Steklov Mat. Inst., 184, 200-207 (in Russian). English translation in: (1994). J. Math. Sci., 63, 540-544.

Móri T.F. and Székely, G.J. (1983). On the Erdos-Renyi generalization of the Borel-Cantelli lemma. Studia Sci. Math. Hunger., 18, 173-182.

Nagaraja, H. N. and David, H. A. (1994). Distribution of the maximum of concomitants of selected ordered statistics, Ann. Statist., 22, 478-494.

Nevzorov, V.B. (1985). On record times and inter-record times for sequences of nonidentically distributed random variables, Zap. Nauch. Sem. POMI(LOMI), 142, 109-118.

Nevzorov, V.B. (1986). Two characterizations using records, Lecture Notes in Mathematics, 1233, Springer, Berlin, 79-85.

Petrov, V.V. (2002). A note on the Borel-Cantelli lemma. Statist. Probab. Lett., 58, $283-286$.

Petrov, V.V. (2004). A generalization of the Borel-Cantelli Lemma. Statist. Probab. Lett., 67, 233-239. 
Pfeifer, D. (1989). Extremal processes, secretary problems and the 1/e law, J. Appl. Probab., 27, 722733 .

Pfeifer, D. (1991). Some remarks on Nevzorovs record model, Adv. Appl. Probab., 23, $823-834$.

Sen, P.K. (1981). Some invariance principles for mixed rank statistics and induced order statistics and some application, Communications in Statistics - Theory and Methods, $10(17)$.

Spitzer, F. (1964). Principles of Random Walk, Van Nostrand, Princeton.

Yang, M.C.K. (1975). On the distribution of the inter-record times in an increasing population, J. Appi. Probab., 12, 148-154. 